\title{
Blob-induced toroidal momentum transport in simple magnetized plasmas
}

\author{
B. Labit, C. Theiler, A. Fasoli, I. Furno and P. Ricci \\ Centre de Recherches en Physique des Plasmas, \\ Ecole Polytechnique Fédérale de Lausanne, \\ Association Euratom-Confédération helvétique, CH-1015 Lausanne
}

\begin{abstract}
The link between toroidal flows and density blobs is experimentally demonstrated in TORPEX simple magnetised plasmas: momentum is transferred from an ideal-interchange mode to density blobs. The phase shift between the toroidal flow and the density perturbations observed in the interchange mode where the blob is born is conserved along the blob radial trajectory. This leads to dipolar structures of the blob-induced flow or to monopolar perturbations, so large that the toroidal flow gets transiently reversed. The turbulent toroidal momentum flux is dominated either by the nonlinear flux or by the convective part but not by the Reynolds stress component.

PACS numbers:
\end{abstract}




\section{INTRODUCTION}

Flows and the associated momentum transport are fundamental components upon the dynamics of accretion disk [1], dynamo effect [2] and turbulent magnetohydrodynamics in the solar wind [3]. The nature of this transport and the origin of the flows in the absence of external momentum sources are crucial questions that remain open to date. In magnetically confined plasmas, recent experimental observations have demonstrated that the spontaneous or intrinsic toroidal rotation profiles cannot be explained with the neoclassical theory of momentum transport only [4-9]. Therefore, in the last decade, several theories have been proposed to identify turbulence driven terms in the toroidal momentum flux. [10-13].

Moreover, experimental observations suggest that the intrinsic plasma rotation is initiated at the edge just inside the last close flux surface (LCFS) and then builds inward [9]. Rotation at the tokamak edge might be determined by scrape-off layer (SOL) field aligned flows which constitute a key mechanism to carry particles and energy towards the divertor plates, regulating recycling and wall damage $[14,15]$. In this region, isolated intermittent structures called blobs, propagating in the radial direction, dominate the cross-field heat and particle transport (see [16] and references therein). Blobs might also carry momentum from the plasma edge into the SOL and could provide a recoil force that might induce a rotation of the core plasma $[10,11]$. While recent numerical simulations $[17,18]$ show that blobs can generate significant flows perpendicular and parallel to the magnetic field, only a single piece of experimental evidence has been reported on the possible link between blob dynamics and field aligned flows. In the JET tokamak SOL, Hidalgo et al [19] have shown that it exists a coupling between the bursty particle transport due to blobs and the parallel Mach number: the larger the transport, the larger the Mach number. However, measurements were limited to two spatial points outside the LCFS and only a statistical analysis could be performed. In addition, Goncalves et al [20] have demonstrated the role of parallel turbulence forces on edge momentum redistribution in the plasma boundary region of the TJ-II stellarator.

In this paper, we investigate the link between toroidal flows and density blobs born from ideal interchange-driven turbulence in toroidal plasmas with open magnetic field lines and with constant curvature. The interchange instability is one of the most common instabilities in magnetized plasmas and is driven by collinear magnetic field curvature and pressure gradient.

Details on the experimental setup and the target plasma are given in Sec. II. Time average profiles of the toroidal flow measured over a large fraction of the cross-section are presented in 
Sec. III. In Sec. IV, we present, for the first time, measurements of 2D time resolved toroidal velocity fluctuations associated with density blobs. The phase shift between the density $n_{e}$ and the toroidal velocity $V_{\phi}$ measured in the region where the blob is born is conserved along its radial propagation. Depending on the experimental conditions, $V_{\phi}$ fluctuations can have a dipolar structure leading to quasi-gaussian statistical properties or a monopolar structure, so large that the toroidal flow is transiently reversed. Finally, we report on first quantitative measurements of a key physics quantity, the turbulent momentum flux, which can be decomposed into three terms: the toroidal Reynolds stress, the convective flux and the nonlinear flux (Sec. V).

\section{EXPERIMENTAL SETUP AND TARGET PLASMA}

The experiments are performed in TORPEX [21], a toroidal device with major and minor radius $\mathrm{R}=1 \mathrm{~m}$ and $\mathrm{a}=0.2 \mathrm{~m}$, a toroidal magnetic field $B_{\phi}$ up to $0.1 \mathrm{~T}$ and a superimposed small vertical magnetic field $B_{z} \leq 4 \mathrm{mT}$ (see Fig. 1a)). This leads to helical magnetic field lines which feature curvature and $\nabla \mathrm{B}$ effects and with both ends terminating on the vacuum vessel with a small pitch angle $\left(\leq 5^{\circ}\right)$. Highly reproducible plasmas are created and sustained for up to $2.5 \mathrm{~s}$ by means of waves in the Electron Cyclotron range of frequencies [22]. In the present study, we focus on the well-established regime in which blobs are born from an interchange wave in $\mathrm{H}_{2}$ plasmas [23, 24]. Following an increase in the pressure gradient, the interchange mode enlarges radially. Since the radial shear of the background vertical $E \times B$ flow is sufficiently large, the mode is sheared-off and a blob is generated [23,24]. The charge separation inside the blob creates an $E \times B$ drift that moves the blob radially outwards [25]. In addition to this usual scenario ( $B_{z}>0$, case $\mathrm{A}$ ), we repeat the same kind of measurements after reversing the vertical magnetic field $\left(B_{z}<0\right.$, case $\left.\mathrm{B}\right)$.

On TORPEX, toroidal flows are measured with a Mach probe which consists of two tungsten circular plates (diameter: $8 \mathrm{~mm}$ ) separated by an insulator and kept perpendicular to the toroidal direction as sketched in Fig 1b). The probe can be moved over a large fraction of the cross-section on a shot-to-shot basis (Fig.1c)). The blob dynamics is not drastically perturbed by the presence of the Mach probe, as revealed by the comparison with data from electrostatic probes of much smaller size [27] and also from visible light fluctuation measurements [28]. While the physical definition of the Mach number is $M_{\phi}(t)=V_{\phi}(t) / c_{s}(t)$, a commonly accepted operational formulation [29] is given by $M(t)=0.4 \log \left[j_{\text {up }}(t) / j_{\text {down }}(t)\right]$ where $V_{\phi}$ is the ion fluid velocity, $c_{s}$ is the

ion sound speed defined as $c_{s} \equiv \sqrt{Z k T_{e} / m_{i}}$ and $j_{\text {up }}$ and $j_{\text {down }}$ are the ion saturation currents 
measured on the two opposed sides of the probe. In TORPEX plasmas, ions are cold $\left(T_{i}<1 \mathrm{eV}\right)$ so $T_{i}$ is neglected with respect to $T_{e}$ in the definition of the ion sound speed $c_{s}$. According to our sign convention, a positive Mach number corresponds to a toroidal flow in the counter-clockwise direction when the torus is seen from top. This is also the direction of the toroidal magnetic field in our experiments.

For both cases $\mathrm{A}$ and $\mathrm{B}$, the time averaged profiles of the plasma density $n_{e}$, the electron temperature $T_{e}$ and the plasma potential $V_{\mathrm{pl}}$ are obtained from Langmuir probe measurements. The first two quantities and the floating potential $V_{\mathrm{fl}}$ are extracted from fits of Langmuir probe characteristics. The plasma potential is deduced from the general formula: $V_{\mathrm{pl}}=V_{\mathrm{fl}}+\mu T_{e}$ where $\mu=3.1 \pm 0.6$ is evaluated experimentally [22]. The time averaged 2D profiles are shown in Fig. 2. Profiles are peaked around $\mathrm{r}=-2 \mathrm{~cm}$ and elongated in the vertical direction. These plasmas are characterized by two distinct poloidal regions with different plasma dynamics: 1) a main plasma region for $-3<r<6 \mathrm{~cm}$ dominated by a coherent interchange wave (frequency $\simeq 4 \mathrm{kHz}$; phase velocity $\simeq 800 \mathrm{~ms}^{-1}$ ); 2) a region on the low-field side for $r>6 \mathrm{~cm}$ with negligible plasma production, dubbed source-free region, characterized by the propagation of plasma blobs, resulting in intermittent transport events [24].

\section{BOUNDARY CONDITIONS AND TIME AVERAGE PROFILES OF THE TOROIDAL FLOW VELOCITY}

The time averaged profiles of the toroidal velocity $V_{\phi}$ are obtained from the Mach number measurements, as shown in Fig. 3. Velocities ranging between 2 and $8 \mathrm{~km} / \mathrm{s}$ are measured, corresponding to $0.1-0.4 \times c_{s}$. The time averaged flow profiles have a more complicated structure than the time averaged plasma pressure, which is slab-like. From a simple 1D picture, neglecting drift terms and assuming a normal incidence of the field lines on the vessel, the plasma is expected to flow at the sound speed $\left(M_{\|}= \pm 1\right)$ at the entrance of the sheath, where the magnetic field lines intersect the vessel and the stagnation point $\left(M_{\|}=0\right)$ should be located at mid distance along the magnetic field line. For the TORPEX magnetic configuration, one would expect $V_{\phi} \rightarrow-c_{s}$ at the bottom of the vessel, $V_{\phi} \sim 0$ at $\mathrm{z}=0$ and $V_{\phi} \rightarrow+c_{s}$ at the top of the vessel for case $\mathrm{A}$ and

the opposite for case B. Qualitatively, the measured profiles are consistent with this picture: $V_{\phi}$ increases with $\mathrm{z}$ for case A and decreases with $\mathrm{z}$ for case B (Fig. 3). Nevertheless, several effects are influencing the time averaged profiles of $V_{\phi}$, such as corrections to the parallel velocity at the 
sheath due to drift $E \times B$ flows perpendicular to the wall: $v_{\|}=c_{s} \pm V_{E \times B} / \tan (\alpha)$ [30]. Here $\alpha$ is the angle formed by the magnetic field line and the vessel. For TORPEX plasmas, even if $V_{E \times B}$ is only about $4 \%$ of $c_{s}$, the angle $\alpha$ is so small $\left(\sim 3^{\circ}\right)$ that the drift term can significantly affect the paralell velocity. These probable effects are supported by an additional test: measurements at $\mathrm{r}=-5$ $\mathrm{cm}$ have been performed with a conducting limiter covering the entire cross-section. In this case, the incidence angle $\alpha$ is close to $\pi / 2$ (i.e. negligible drift effects) and the distance along the magnetic field lines between the limiter and the measurement plane is identical along $\mathrm{z}$ for magnetic field lines with both ends terminating on the limiter (see Fig. 4a)). As expected with this setup, the profile of the toroidal Mach number is constant along $\mathrm{z}$ for magnetic field lines terminating on both ends on the limiter (Fig. 4b)).

\section{PROPERTIES OF MACH NUMBER FLUCTUATIONS IN THE BLOB REGIME}

Intermittent density fluctuations are characterized by strongly non-Gaussian probability density functions (PDF) (see [31] and references therein). The third moment of these PDF, the skewness, is clearly not equal to zero. This is illustrated in Fig. 5a), where the radial profiles of the skewness for the ion saturation current and the Mach number are plotted for both cases, A and B. Similarly to measurements across the LCFS in tokamaks [32], the $I_{\text {sat }}$ skewness goes from negative values, indicating the region where the blobs are born $(\mathrm{r} \sim-3 \mathrm{~cm})$, to positive values, where the blobs propagate radially $(r>6 \mathrm{~cm})$. For the Mach number, even if the time average profiles are different for cases $\mathrm{A}$ and $\mathrm{B}$, the skewness profiles are rather similar. It is worth noting that the skewness of the Mach number is almost always negative and tends to zero in the source-free region where blobs propagate. In this region, the Mach number PDF is more Gaussian than the density PDF, an observation that is in agreement with previous results from the JET tokamak [19]. The joint probability between $M_{\phi}$ and $I_{\text {sat }} / \sigma$, where $\sigma$ is the standard deviation of $I_{\text {sat }}$, computed from signals measured at $\mathrm{r}=15 \mathrm{~cm}, \mathrm{z}=0 \mathrm{~cm}$ (case A) indicates that the most probable toroidal Mach number doesn't change when plasma density bursts $\left(I_{\text {sat }} / \sigma \geq 2\right)$ are detected (Fig. 5b)). At a first sight, this seems to suggest that the toroidal Mach number is not affected by the blob propagation in the source-free region.

To clarify this issue, we look at the plasma dynamics over the cross-section using the conditional average (CA) technique [34]. The CA technique is a well establish method to detect coherent features in turbulence and relies on a good reproducibility of the discharges, which is the case in 
simple toroidal plasmas. It is based on a reference probe which is kept fixed in position and another probe that can be moved in the plasma. In our case, the analysed data are taken from the Mach probe which is moved on a shot-to-shot basis in order to cover a large fraction of the cross-section and the reference signal $I_{\text {ref }}$ is measured, for each shot, with a standard Langmuir probe positioned in the source-free region. For each shot, peaks in the $I_{\text {ref }}$ time trace, interpreted to be due to the propagation of a blob in front of the probe, are detected with the condition $I_{\text {ref }}>3.5 \sigma_{\text {ref }}$ where $\sigma_{\text {ref }}$ is the standard deviation of the reference signal. Approximately 350 events are detected in a $1 \mathrm{~s}$ duration discharge. Time intervals around the detected peaks are sampled in the Mach number time series and the ensemble average over these intervals is performed to obtain the conditional average. At the end of the process, one can reconstruct the $2 \mathrm{D}$ ensemble-average time evolution $(-300 \mu s<\tau<300 \mu s)$ of the Mach number around an averaged triggering event at $\tau=0$. The duration of the time evolution reconstruction is limited to the duration of the coherent event, here the ejection and the propagation of the blob. In the data from the movable probe, we are not specifically looking at large events as in the reference signal, so it might be possible to capture lower fluctuations and if there are coherent with the trigger events, they will remain after the ensemble average, otherwise they will be smeared out.

For case A, both the ion saturation current and the toroidal Mach number are conditionally sampled. The reference signal is the ion saturation current $I_{\text {ref }}$ measured by a Langmuir probe located at $\mathrm{r}=13 \mathrm{~cm}$ and $\mathrm{z}=0 \mathrm{~cm}$ and toroidally spaced by $\Delta \Phi=90^{\circ}$. The spatial resolution is $2 \mathrm{~cm}$ in both directions, radial and vertical. The results, shown in Fig. 6, represent the CA fluctuations of $I_{\text {sat }}$ and $M_{\phi}$ over a large fraction of the cross section. Few tens of microseconds before the blob is detected on the reference probe ( $\tau=-160 \mu \mathrm{s}$; Fig. 6a-b)), the interchange mode located around r=-2 cm is visible for $\tilde{I}_{\text {sat }}$ and for $\tilde{M}_{\phi}$. The mode for $\tilde{M}_{\phi}$ is already elongated in the radial direction and is delayed with respect to the density fluctuations with a phase $\angle\left(\tilde{I}_{\text {sat }}, \tilde{M}_{\phi}\right) \simeq \pi / 2$. When the triggering event is detected on the reference probe $(\tau=0 \mu \mathrm{s}$, Fig. 6(c-d)), the density blob, which is a filamentary structure aligned with the magnetic field line, is not yet totally sheared-off from the positive wave crest. Almost no fluctuations in the toroidal Mach number are detected at the peak of the density blob $(\mathrm{r}=13 \mathrm{~cm}, \mathrm{z}=-5 \mathrm{~cm})$, consistent with the absence of visible correlation in the joint probability. Nevertheless, a positive fluctuation (blob-like) and a negative fluctuation (holelike) in $\tilde{M}_{\phi}$ are visible at different vertical positions in the cross-section (z=-8 cm and $\mathrm{z}=-2 \mathrm{~cm}$, respectively). This corresponds to the same phase difference observed in the mode region. This displacement in the fluctuation locations is larger than the spatial uncertainty associated with the 
$2 \mathrm{~cm}$ resolution of the measurements. This is confirmed by the measurements at higher resolution which are presented in the next section. The toroidal Mach number is affected by the blob induced perturbation but not at the location of the blob itself, revealing a dipolar structure. This emphasizes the importance of 2D measurements of plasma flows that are up to now only possible in basic plasma devices and not in the tokamak SOL. For case B, the observed phase in the mode region between the toroidal flow and the density is $\angle\left(\tilde{I}_{\text {sat }}, \tilde{M}_{\phi}\right)=\pi$. Although there is insufficient data from case B to generate a counterpart to Fig. 6, it will be demonstrated in the next section that the density blob is associated with a toroidal Mach number hole in the source-free region.

\section{BLOB INDUCED TOROIDAL MOMENTUM TRANSPORT}

Spatio-temporal correlation between $M_{\phi}$ and $I_{\text {sat }}$ is demonstrated in the blob scenario in TORPEX. However, this does not necessarily imply a similar correlation between density and toroidal velocity. Indeed, it has been shown that a density blob is significantly hotter than the surrounding plasma [33]. Therefore, fluctuations in the toroidal Mach number might be due also to $c_{s}\left(T_{e}\right)$ fluctuations. To quantify this effect, we use a combined version of the conditional averaging (CA) and boxcar techniques [24]. This technique is explained in details in [23] and might be summarized as follows: a reference probe, fixed in space in the blob region measures the ion saturation current $I_{\text {ref }}$. In this time trace, we are detecting spikes defined by their amplitude which are the signature of a density blob passing by the reference probe. To each time a blob is detected corresponds one measured current $I$ and one applied voltage $V$ on Mach probe tips. Since blob events are not correlated with the swept voltage applied to the probe, the ensemble of currents and voltages gives a Langmuir characteristics which can be fitted to estimate plasma parameters. This exercise is repeated at different time lags around the time of blob detection. Here, with approximately 900 detected triggers, we are reconstructing the time evolution $(-300 \mu \mathrm{s}<\tau<+300 \mu \mathrm{s})$ of the den-

sity, temperature, plasma potential and toroidal Mach number measured at the same location and correlated with a blob detected at $\tau=0$ on a reference probe [26].

For both cases $\mathrm{A}$ and $\mathrm{B}$, we perform a vertical scan of the Mach probe between $-7 \leq z \leq 6$ $\mathrm{cm}$ at $\mathrm{r}=12.5 \mathrm{~cm}$ with a spatial resolution of $1 \mathrm{~cm}$. For each probe location, three $2.5 \mathrm{~s}$ duration plasma discharges are recorded and the threshold condition on the signal measured by the reference probes is set to $I_{\text {ref }}>3.5 \sigma$. These probes are toroidally spaced by $90^{\circ}$ from the Mach probe and are positioned at three different vertical locations: $\left(r_{\mathrm{ref}}, z_{\mathrm{ref}}\right)^{A}=(12,+[3.5,5.5,8.5]) \mathrm{cm}$ for 
case A and $\left(r_{\text {ref }}, z_{\text {ref }}\right)^{B}=(12,-[3.5,5.5,8.5]) \mathrm{cm}$ for case B (Fig. 7a-b)). When $B_{z}$ is reversed, the reference probe position is changed to ensure that field aligned blobs are still detected in the vertical range covered by the Mach probe. The results shown in Fig. 7 represent the vertical profiles of the plasma density $n_{e}$ (c-d) and the toroidal velocity $V_{\phi}(\mathrm{e}-\mathrm{f})$ for the three reference probe positions. Color profiles are averaged over $20 \mu$ s around $\tau_{b}$, where $\tau_{b} \simeq 20 \mu \mathrm{s}$ is the time at which the pressure blob is detected on the Mach probe (a positive $\tau_{b}$ is consistent with the relative radial position of the Mach probe with respect to the reference probe). These profiles have to be compared with the time average profiles (thin black) computed in the absence of blobs $(-300 \mu \mathrm{s}$ $<\tau<-100 \mu$ s) and independent of the trigger position.

In the Mach probe plane, density blobs correspond to the maximum density in the vertical profiles. Since blobs are field aligned structures, they should be detected at $z_{b} \simeq z_{\text {ref }}^{A}-5.5 \mathrm{~cm}$ for case $\mathrm{A}$ and at $z_{b} \simeq z_{\text {ref }}^{B}+5.5 \mathrm{~cm}$ for case $\mathrm{B}$ where $5.5 \mathrm{~cm}$ is a quarter of the vertical displacement of the magnetic field line after one complete turn. The observed locations of the maxima are in good agreement with this predicition for case A (Fig. 7c)), while the agreement is not as good for case B (Fig. 7d)). For case A, $V_{\phi}$ decreases by about $20 \%$ at $z=z_{b}$ and a phase shift reminescent of the blob origin is evident: largest positive and negative fluctuations (up to $\pm 50 \%$ with respect to the unperturbed profile) are localised at $z=z_{b} \mp 4 \mathrm{~cm}$, revealing again the dipolar structure that reduces the skewness towards zero. The largest positive and negative perturbations are of the same order of magnitude. For case B, a density blob is rather associated with a hole for the toroidal velocity. This is reminiscent of the phase in the interchange mode region. The density blob induces such a large perturbation that the toroidal flow is transiently reversed. This might have strong implications for the impurity transport in a tokamak SOL.

A key physics quantity required for a comparison between experimental observations and theoretical predictions is the turbulent momentum flux which can be written as follows [13]:

$$
\Pi_{r, \phi}=\left\langle n^{0}\right\rangle\left\langle v_{r}^{1} V_{\phi}^{1}\right\rangle+\left\langle v_{r}^{1} n^{1}\right\rangle\left\langle V_{\phi}^{0}\right\rangle+\left\langle n^{1} v_{r}^{1} V_{\phi}^{1}\right\rangle
$$

Steady-state terms in the absence of blobs are $n^{0}$ and $V_{\phi}^{0}$ and blob induced contributions are $n^{1}, V_{\phi}^{1}$ and $v_{r}^{1}$. In the source-free region, the fluctuating radial velocity $v_{r}^{1}$ is essentially determined by the local radial $V_{E \times B}$ [33] (Fig. 7g-h)). The first term in Eq.(1) is the toroidal Reynolds stress (RS), the second is the convective flux and the third is the nonlinear flux which might be driven by modemode coupling or turbulence spreading. Vertical profiles of these three terms for $z_{\text {ref }}^{A}=+5.5 \mathrm{~cm}$ and for $z_{\text {ref }}^{B}=-5.5 \mathrm{~cm}$ are plotted in Fig. 8a-b). For case A, the transport of toroidal momentum 
is essentially convective. The Reynolds stress and the nonlinear term are negligible. For case B, the nonlinear term has significantly increased compared to case A suggesting strongly nonlinear precesses at work in generating rotation. This term slightly dominates over the convective term and the RS term is still negligible.

The number of triggering events is sufficiently large to allow similar analysis for various blob amplitudes. Blob amplitude is defined in $\sigma$ units where $\sigma$ is the standard deviation of the reference signal. The overall picture doesn't change significantly, in particular the phase shift observed in the mode remains almost unchanged with the blob amplitude. For both cases, the largest positive, $\max \left(V_{\phi}^{1}\right)$, and negative, $\min \left(V_{\phi}^{1}\right)$, blob-induced toroidal flows are represented in Fig. 9. Both $\max \left(V_{\phi}^{1}\right)$ and $\min \left(V_{\phi}^{1}\right)$ increase with the blob size. For case $\mathrm{A},\left|\min \left(V_{\phi}^{1}\right)\right| \sim \max \left(V_{\phi}^{1}\right)$ consistent with statistical properties close to gaussianity, while for case $\mathrm{B},\left|\min \left(V_{\phi}^{1}\right)\right|$ is larger than $\max \left(V_{\phi}^{1}\right)$. The positive $V_{\phi}^{1}$ being observed few tens of microseconds before the blob is detected in that case. It has to be noted that the scaling with the blob amplitude is different between case A and case B.

\section{CONCLUSION}

In summary, toroidal flows have been measured in TORPEX in the blob regime scenario in $\mathrm{H}_{2}$ plasmas for $B_{z}>0$ (case A) and $B_{z}<0$ (case B). Their time average 2D profiles seem to be determined mainly by the boundary conditions and by $E \times B$ drift velocity effects. We have presented, for the first time, measurements of 2D time resolved toroidal velocity fluctuations associated with density blobs. Time resolved measurements of the blob-induced toroidal flow $V_{\phi}^{1}(\underline{\mathrm{r}}, \tau)$ lead to the following conclusions: 1) The phase shift between $n_{e}^{1}$ and $V_{\phi}^{1}$ is conserved along the blob trajectory from the interchange mode region where it is born to the far source-free region where the blob is dissipated; 2) For case A, the perturbed flow shows a dipolar structure leading to statistical properties close to gaussianity; the turbulent momentum flux is dominated by the convective term; 3 ) For case $\mathrm{B}, V_{\phi}^{1}$ is a monopolar structure which can be so large that the toroidal flow gets transiently reversed when the blob propagates radially; the nonlinear term dominates the toroidal momentum flux. The essential difference between cases $\mathrm{A}\left(B_{\phi}>0, B_{z}>\right.$ $0)$ and $\mathrm{B}\left(B_{\phi}>0, B_{z}<0\right)$ sets in the phase shift between density fluctuations and toroidal velocity fluctuations observed in the ideal interchange mode. We have demonstrated that the difference in phase shift is responsible for differences in toroidal momentum transport once the blob is formed. Addressing the physics at the origin of the phase shift between the interchange mode and the 
toroidal flow is beyond the scope of this paper. However, we stress that case A and B are not identical: For both cases, in the mode region, the large $E \times B$ flow is directed upward. By reversing the vertical magnetic field $B_{z}$, we change the incidence angle at the top and the bottom of the vessel with respect to the $E \times B$ flow. So, both configurations are not strictly speaking symmetrical. The differences in the time averaged profiles of the toroidal flow and in the phase in the interchange mode induced by the reversal of $B_{z}$ are not entirely understood up to now. Similar measurements in the presence of a conducting poloidal limiter will clarify our understanding.

The present results demonstrate unambiguously the link between pressure-driven turbulent structures and plasma flows: momentum is transferred from the ideal-interchange mode to the blobs and lost from the mode region, providing a recoil force that can be a source of intrinsic rotation for systems with closed magnetic field lines. Therefore, these results are of interest for a variety of plasmas, which are prone to interchange instabilities, such as in tokamaks, in reversed field pinches (RFP), in magnetic dipoles, and in simple magnetized tori. Our findings also highlight the importance of boundary conditions and drifts for 3D global simulations of plasma turbulence and provide a reference case for theory and simulation to improve our understanding of the physics underlying the access to improved confinement regimes in magnetic fusion devices.

This work is partly funded by the Swiss National Science Foundation. P.R. was supported by a EURATOM fusion fellowship.

[1] B. Coppi and P.S. Coppi, 2001, Phys. Rev. Lett. 87051101

[2] N. Leprovost and E.-J. Kim, 2008, Phys. Rev. Lett. 100, 144502

[3] W-C. Müller and D. Biskamp, 2000, Phys. Rev. Lett. 84, 475

[4] L.-G. Eriksson, E. Righi and K.-D. Zastrow, 1997, Plasma Phys. Controlled Fusion 39, 27.

[5] J. E. Rice, A. Ince-Cushman, J.S. deGrassie, L.-G. Eriksson, Y. Sakamoto, A. Scarabosio, A. Bortolon, K.H. Burrell, B.P. Duval, C. Fenzi-Bonizec, M.J. Greenwald, R.J. Groebner, G.T. Hoang, Y. Koide, E.S. Marmar, A. Pochelon and Y. Podpaly, 2007, Nucl. Fusion 47, 1618.

[6] B. P. Duval, A. Bortolon, A. Karpushov, R. A. Pitts, A. Pochelon, O. Sauter, A. Scarabosio, and G. Turri, 2008, Plasma Phys. 15, 056113.

[7] M. Yoshida, Y. Kamada, H. Takenaga, Y. Sakamoto, H. Urano, N. Oyama, and G. Matsunaga, 2008, Phys. Rev. Lett. 100, 105002. 
[8] W. M. Solomon, K.H. Burrell, A.M. Garofalo, A.J. Cole, R.V. Budny, J.S. deGrassie, W.W. Heidbrink, G.L. Jackson, M.J. Lanctot, R. Nazikian, H. Reimerdes, E.J. Strait, M.A. Van Zeeland and the DIII-D Rotation Physics Task Force, 2009, Nucl. Fusion 49, 085005.

[9] J. S. deGrassie, R.J. Groebner, K.H. Burrell and W.M. Solomon, 2009, Nucl. Fusion 49, 085020.

[10] B. Coppi, D.A. D’Ippolito, S.I. Krasheninnikov, M. Lontano, J.R. Myra, P. Nataf, D.A. Russell, 2006, Proc. of the 33rd EPS Conference, Rome, 2006, O4.017.

[11] B. Coppi, 2002, Nucl. Fus., 421.

[12] J. S. deGrassie, 2009, Plasma Phys. Control. Fusion 51124047

[13] P. H. Diamond, C.J. McDevitt, Ö.D. Gürcan, T.S. Hahm, W. X. Wang, E.S. Yoon, I. Holod, Z. Lin, V. Naulin and R. Singh, 2009, Nucl. Fus. 49, 045002.

[14] W. Fundamenski, R.A. Pitts and JET EFDA contributors, 2006, Plasma Phys. Controlled Fusion 48 109

[15] A. Loarte, B. Lipschultz, A.S. Kukushkin, G.F. Matthews, P.C. Stangeby, N. Asakura, G.F. Counsell, G. Federici, A. Kallenbach, K. Krieger, A. Mahdavi, V. Philipps, D. Reiter, J. Roth, J. Strachan, D. Whyte, R. Doerner, T. Eich, W. Fundamenski, A. Herrmann, M. Fenstermacher, P. Ghendrih, M. Groth, A. Kirschner, S. Konoshima, B. LaBombard, P. Lang, A.W. Leonard, P. Monier-Garbet, R. Neu, H. Pacher, B. Pegourie, R.A. Pitts, S. Takamura, J. Terry, E. Tsitrone and the ITPA Scrape-off Layer and Divertor Physics Topical Group, 2007, Nucl. Fus., 47 S203

[16] S. J. Zweben, J. A. Boedo, O. Grulke, C. Hidalgo, B. LaBombard, R. J. Maqueda, P. Scarin and J. L. Terry, 2007, Plasma Phys. Control. Fusion 49 S1.

[17] J. R. Myra, D. A. Russell, and D. A. D’Ippolito, 2008, Phys. Plasmas 15, 032304.

[18] A. Yu. Pigarov, S. I. Krasheninnikov, B. LaBombard and T. D. Rognlien, 2008, Contrib. Plasma Phys. 4882.

[19] C. Hidalgo, B. Gonçalves, C. Silva, M. A. Pedrosa, K. Erents, M. Hron, and G. F. Matthews, 2003, Phys. Rev. Lett. 91, 065001.

[20] B. Gonçalves, C. Hidalgo, M. A. Pedrosa, R. O. Orozco, E. Sànchez and C. Silva 2006, Phys. Rev. Lett. 96, 145001

[21] A. Fasoli, B. Labit, M. McGrath, S. H. Müller, G. Plyushchev, M. Podestà, and F. M. Poli, 2006, Phys. Plasmas 13055903.

[22] M. Podestà, A. Fasoli, B. Labit, M. McGrath, S. H. Müller and F. M. Poli, 2005, Plasma Phys. Control. Fusion 47, 1989. 
[23] I. Furno, B. Labit, A. Fasoli, F. M. Poli, P. Ricci, C. Theiler, S. Brunner, A. Diallo, J. P. Graves, M. Podestà and S. H. Müller, 2008, Phys. Plasmas, 15, 055903.

[24] I. Furno, B. Labit, M. Podestà, A. Fasoli, S. H. Müller, F. M. Poli, P. Ricci, C. Theiler, S. Brunner, A. Diallo, and J. Graves, 2008, Phys. Rev. Lett., 100, 055004.

[25] C. Theiler, I. Furno, P. Ricci, A. Fasoli, B. Labit, S. H. Müller, and G. Plyushchev, 2009, Phys. Rev. Lett., 103, 065001.

[26] B. Labit, I. Furno, M. Podestà and A. Fasoli, 2008, Rev. Sci. Instrum. 79, 086104.

[27] C. Theiler, I. Furno, A. Kuenlin, P. Marmillod and A. Fasoli, 2011, Rev. Sci. Instrum. 82, 013504

[28] D. Iraji, I. Furno, A. Fasoli and C. Theiler, 2010, Phys. Plasmas 17, 122304.

[29] K-S. Chung, I. H. Hutchinson, B. LaBombard, and R. W. Conn, 1989, Phys. Fluids B 1, 2229.

[30] R.H.Cohen and D. D. Ryutov, 1995, Com. Plas. Phys. Contr. Fus., 16, 255.

[31] B. Labit, I. Furno, A. Fasoli, A. Diallo, S. H. Müller, G. Plyushchev, M. Podestà, and F. M. Poli, 2007, Phys. Rev. Lett. 98255002.

[32] J. A. Boedo, D. L. Rudakov, R. A. Moyer, G. R. McKee, R. J. Colchin, M. J. Schaffer, P. G. Stangeby, W. P. West, S. L. Allen, T. E. Evans, R. J. Fonck, E. M. Hollmann, S. Krasheninnikov, A. W. Leonard, W. Nevins, M. A. Mahdavi, G. D. Porter, G. R. Tynan, D. G. Whyte, and X. Xu, 2003, Phys. Plasmas 101670.

[33] M. Podestà, A. Fasoli, B. Labit, I. Furno, P. Ricci, F.M. Poli, A. Diallo, S.H. Müller, and C. Theiler, 2008, Phys. Rev. Lett. 101, 045001.

[34] H. Johnsen, H. L. Pécseli, and J. Trulsen, 1987, Phys. Fluids 30, 2239. 

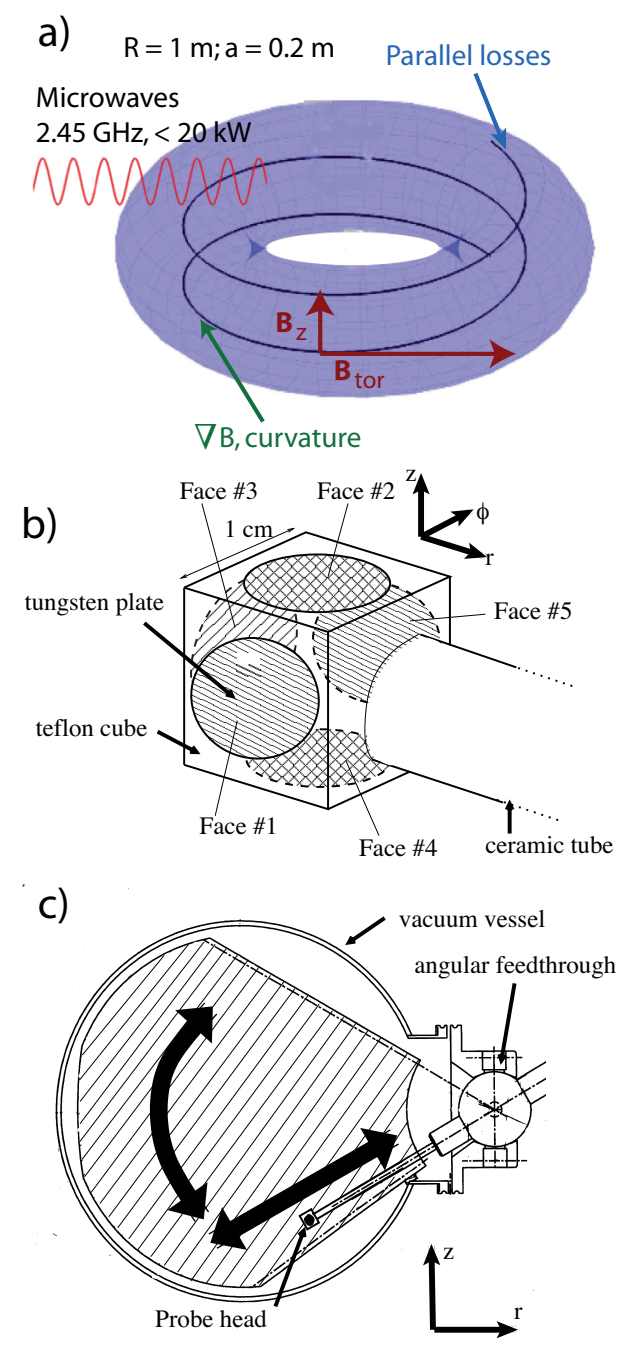

FIG. 1: (Color online) a) Schematical view of the TORPEX geometry with a helical magnetic field line. b) Sketch of the Mach probe head installed on TORPEX. Pins \#1 and \#5 are perpendicular to the toroidal direction. c) Schematic of the probe mounted on a movable system. 

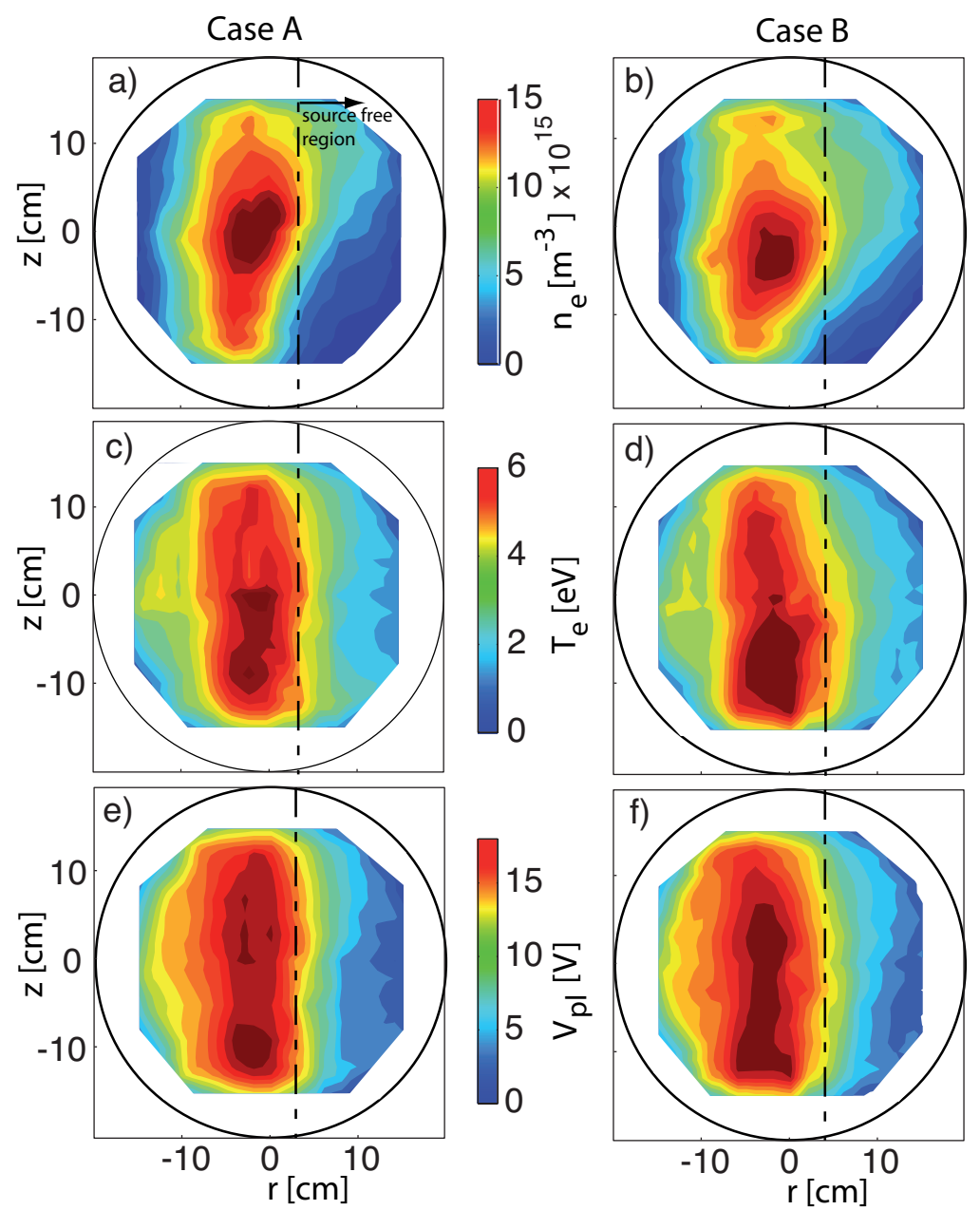

FIG. 2: (Color online) Time average profiles of the density (a-b), the electron temperature (c-d) and the plasma potential (e-f) for $B_{z}>0$ (left column) and $B_{z}<0$ (right column). The vertical dashed black line indicates the free-source regions. 

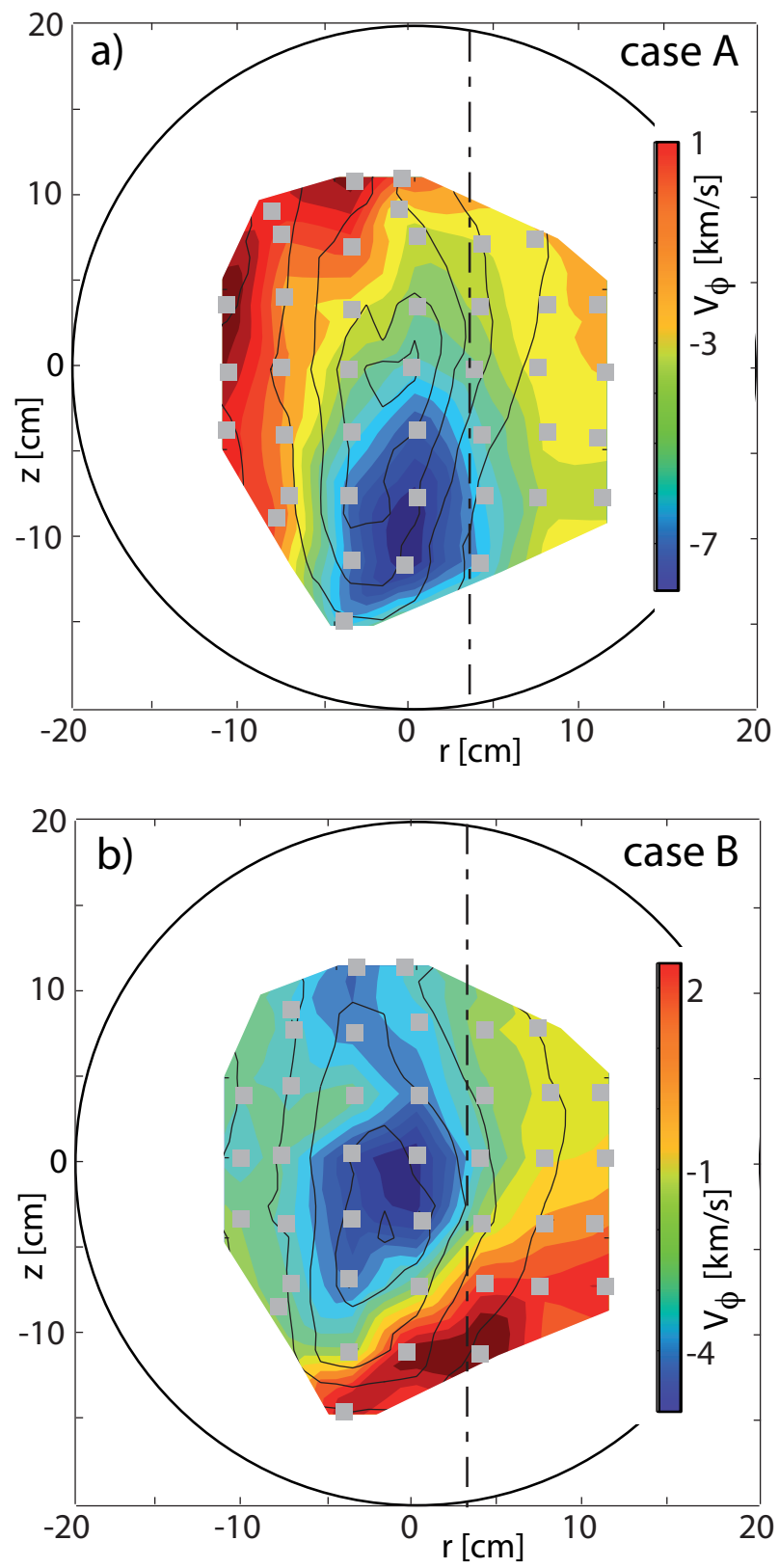

FIG. 3: (Color online) Time average profiles of the toroidal velocity $\bar{V}_{\phi}$ measured over the poloidal crosssection for a) the case $\mathrm{A}\left(B_{\phi}>0, B_{z}>0\right)$ and b) the case $\mathrm{B}\left(B_{\phi}>0, B_{z}<0\right)$. Positive velocities mean that the plasma flows counter-clockwise when the torus is seen from top. The black contours indicate the plasma pressure $\bar{p}_{e}$ and the black dashed vertical line indicates the source free region. Each gray square represents the Mach probe position on a shot-to-shot basis with its size to scale with respect to the vacuum vessel. 


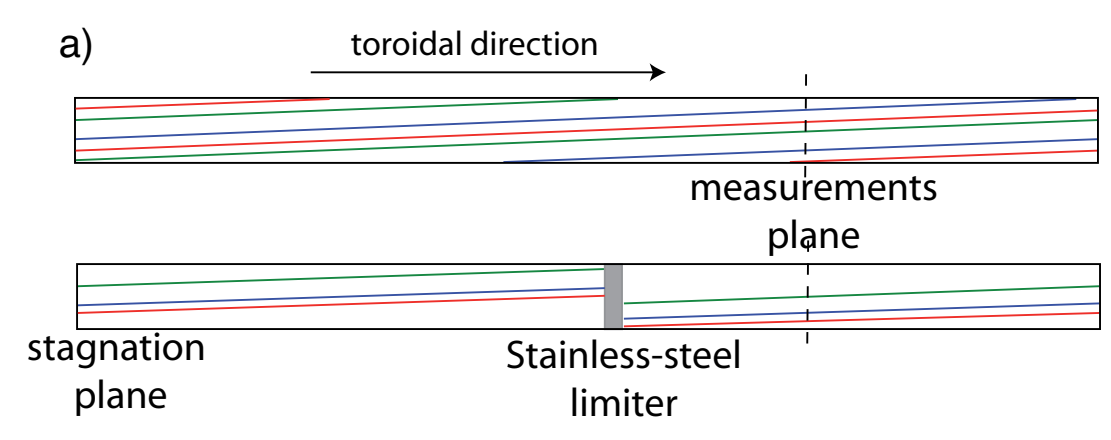

b)

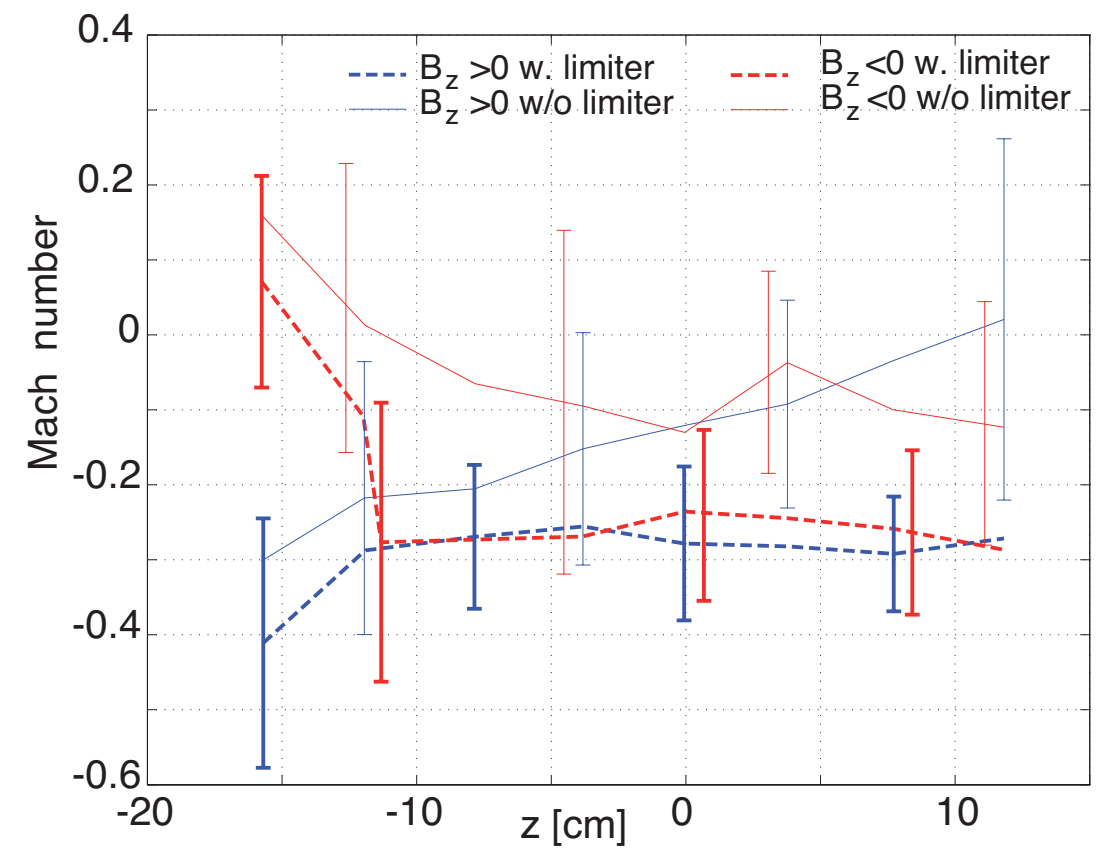

FIG. 4: (Color online) a) Unwrapped torus at a given radial position showing how the distance from the measurement plane to the wall changes in presence of a stainless-steel limiter. For clearness, the pitch angle of the magnetic field line is exaggerated compared to the actual experiments. It corresponds roughly to $B_{z} \sim 36 \mathrm{mT}$; b) Time average profile of the Mach number in the vertical direction at $\mathrm{r}=-5 \mathrm{~cm}$ for cases A and B with (thick lines) and without (thin lines) the limiter. Error bars are given by the standard deviation of the Mach number time series. 

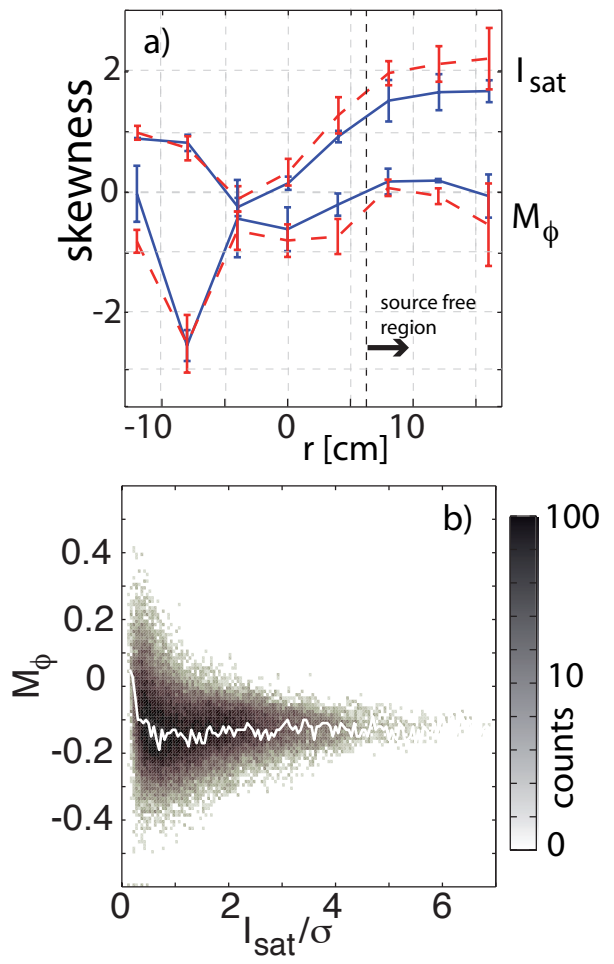

FIG. 5: (Color online) a) Radial profiles averaged between $\mathrm{z}=-6 \mathrm{~cm}$ and $\mathrm{z}=6 \mathrm{~cm}$ of the skewness for $I_{\mathrm{sat}}$ and $M_{\phi}$ for case A (solid blue) and case B (dashed red). Error bars are given by the standard deviation of the skewness estimated at different vertical positions. b) Joint probability between $I_{\text {sat }} / \sigma$ and $M_{\phi}$ at $\mathrm{r}=15$ $\mathrm{cm}, \mathrm{z}=0 \mathrm{~cm}$ for case A. The white line indicates the highest $M_{\phi}$ probability for a given value of $I_{\text {sat }} / \sigma . \sigma$ is the standard deviation of $I_{\text {sat }}$. 


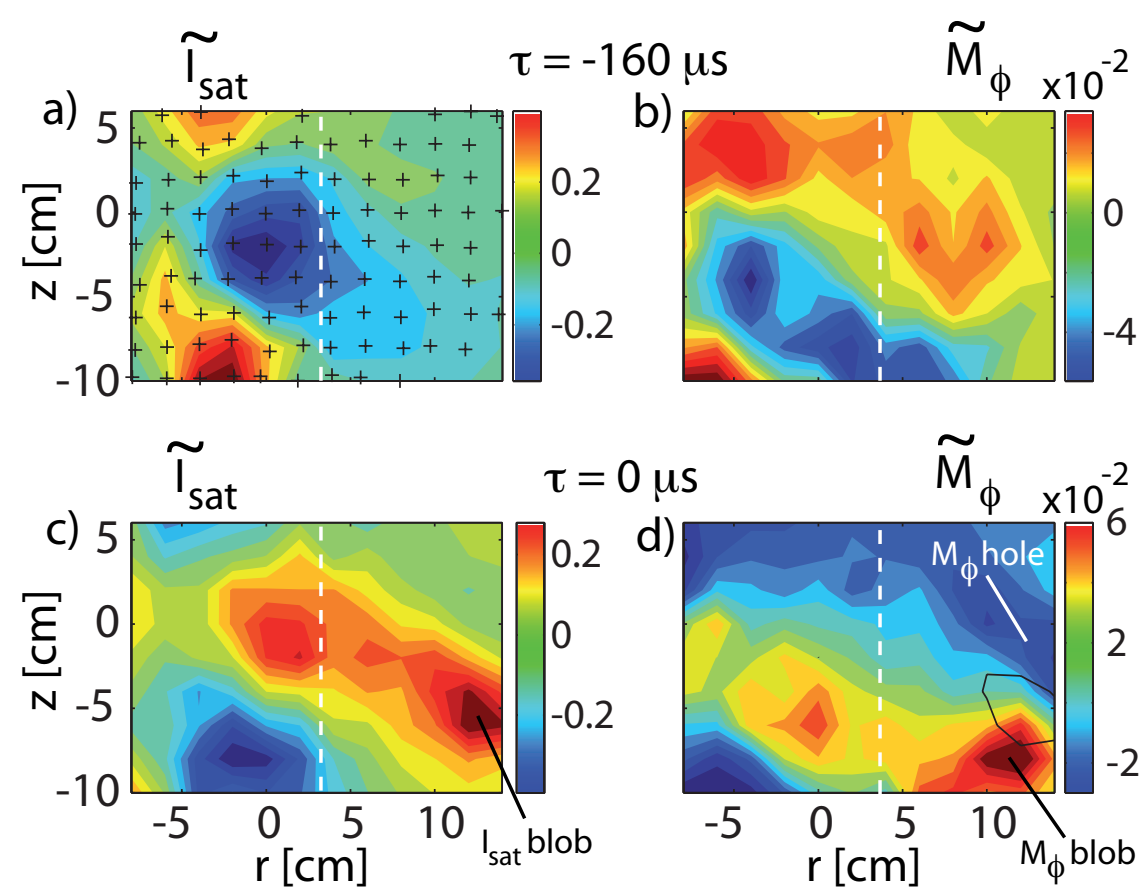

FIG. 6: (Color online) For case A, 2D snapshots of fluctuations of the ion saturation current (left column) and of the toroidal Mach number (right column) reconstructed from conditional average sampling at $\tau=$ $-160 \mu s$ (top row) and $\tau=0 \mu s$ (bottom row). The measurement points are represented by the crosses in subplot a). To guide the eyes, the density blob is added on subplot d) (black contour). The white vertical dashed line separates the source region on the left where the interchange wave propagates upwards from the source-free or blob region on the right (enhanced online). 

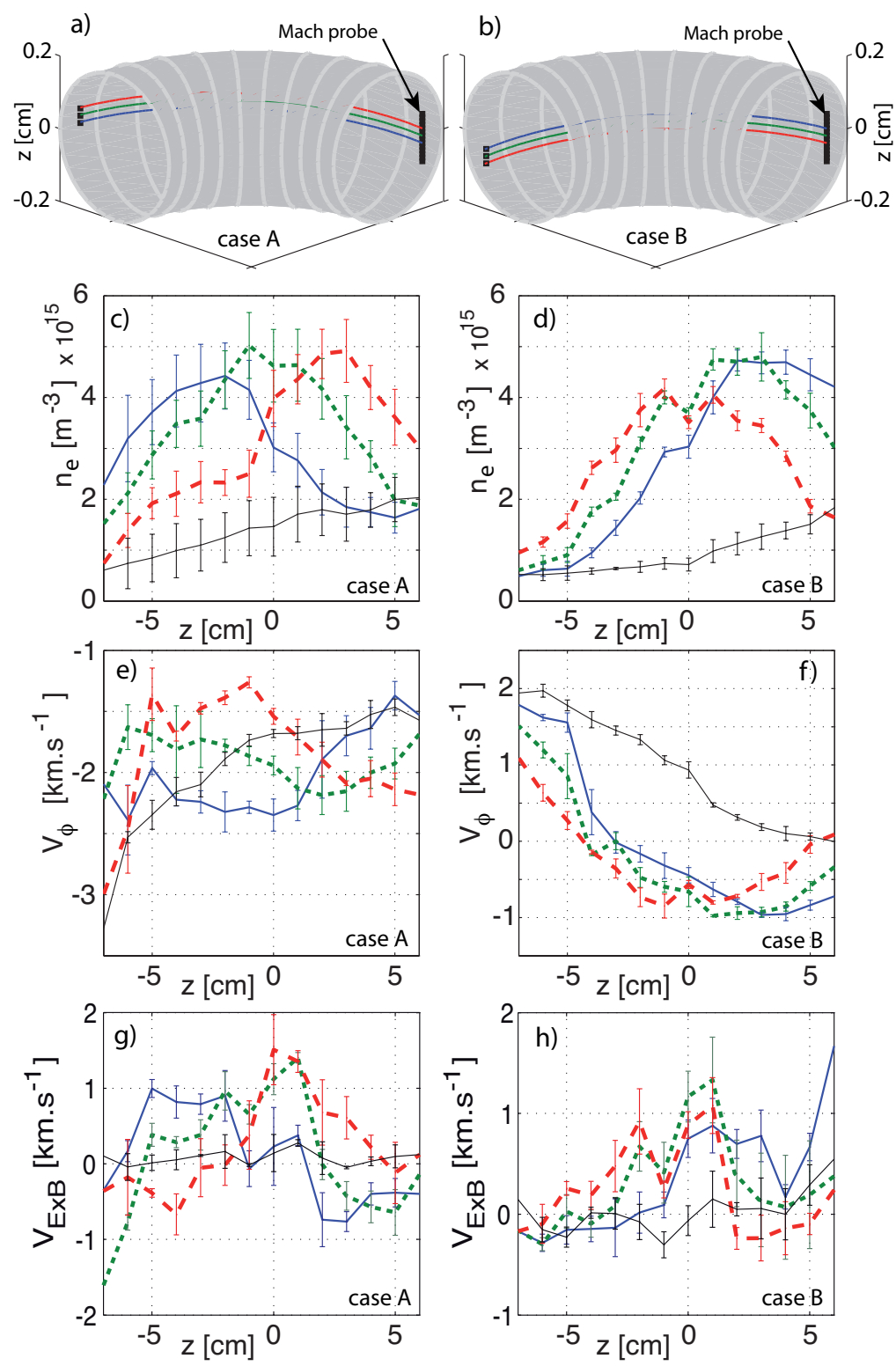

FIG. 7: (Color online) a-b) Positions of the Mach probe in the blob region and positions of the reference probe toroidally separated by $90^{\circ}$. To guide the eyes, field lines intercepting the reference probes are plotted. For clearness, the major radius of the torus is halved. Vertical profiles of the quantities after CA for 3 reference signals at $r_{\text {ref }}^{A, B}=12 \mathrm{~cm}, z_{\text {ref }}^{A}=+2.5 \mathrm{~cm}, z_{\text {ref }}^{B}=-2.5 \mathrm{~cm}$ (solid, blue), $z_{\text {ref }}^{A}=+5.5 \mathrm{~cm}$, $z_{\text {ref }}^{B}=-5.5 \mathrm{~cm}$ (dotted, green), $z_{\text {ref }}^{A}=+8.5 \mathrm{~cm}, z_{\text {ref }}^{B}=-8.5 \mathrm{~cm}$ (dashed, red) cm: c-d) density, e-f) toroidal velocity, g-h) radial E $\times$ B velocity for case A (left column) and case B (right column). For subplots $\mathrm{c}-\mathrm{h})$, time averaged profiles computed in the absence of blobs $(-300 \mu \mathrm{s}<\tau<-100 \mu \mathrm{s})$ are shown with a thin black line. Error bars are given by the standard deviation of the plasma parameters estimated for three identical shots. 

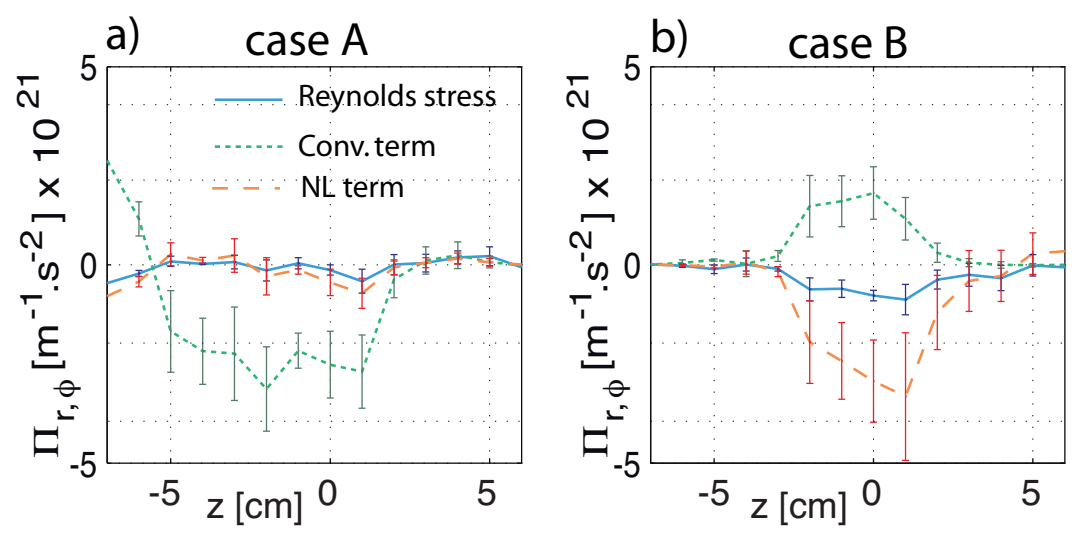

FIG. 8: (Color online) Vertical profiles of the three terms of the toroidal momentum flux given in Eq. 1 for $z_{\text {ref }}^{A}=+5.5 \mathrm{~cm}$ and $z_{\text {ref }}^{B}=-5.5 \mathrm{~cm}$. Error bars are given by the standard deviation of the plasma parameters computed for three identical shots.
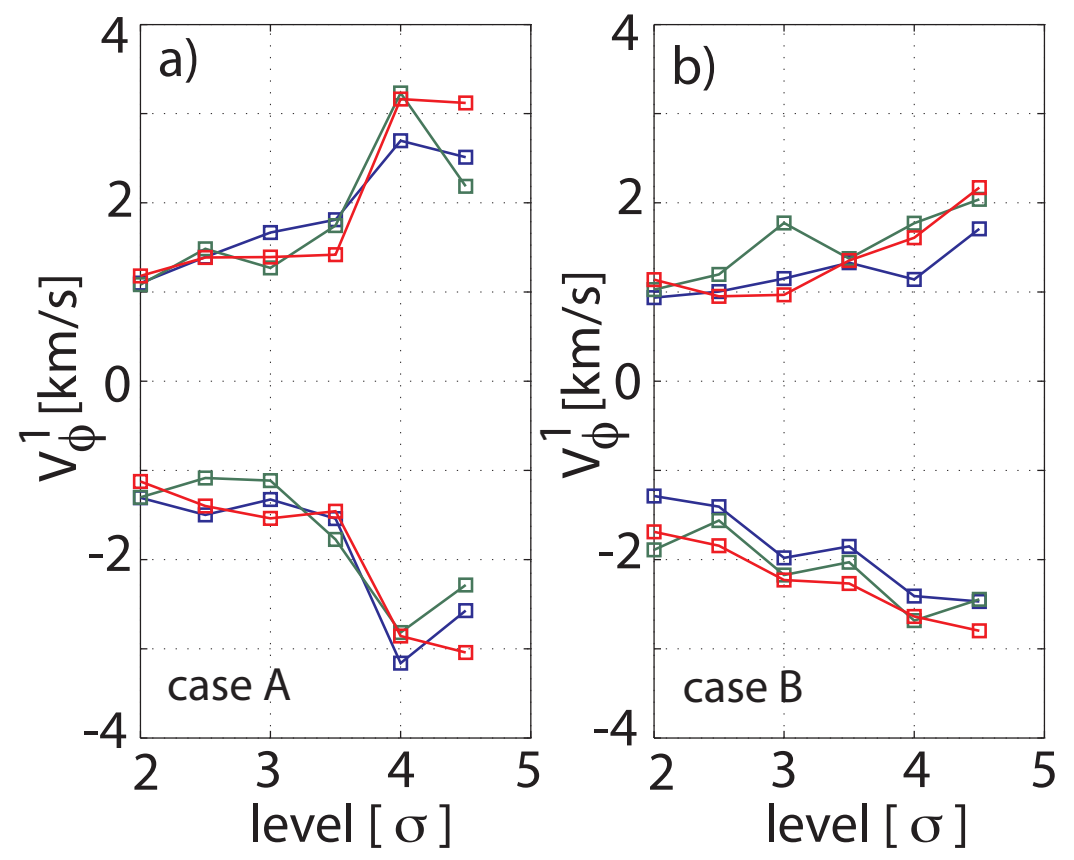

FIG. 9: (Color online) Minimum and maximum toroidal velocity fluctuations as a function of the blob amplitude a) for case A and b) for case B. The analysis is performed for 3 reference probe locations. Blob amplitude is defined in $\sigma$ units where $\sigma$ is the standard deviation of the reference signal. 